\title{
Development and Characterization of MDR1 (Mdr1a/b) CRISPR/Cas9 Knockout Rat Model
}

\author{
Chenmeizi Liang, ${ }^{1}$ Junfang Zhao, ${ }^{1}$ Jian Lu, ${ }^{1}$ Yuanjin Zhang, Xinrun Ma, Xuyang Shang, \\ Yongmei Li, Xueyun Ma, Mingyao Liu, and Xin Wang
}

Shanghai Key Laboratory of Regulatory Biology, Institute of Biomedical Sciences and School of Life Sciences, East China Normal University, Shanghai, People's Republic of China (C.L., J.Z., J.L., Y.Z., Xi.M., X.S., Y.L., Xu.M., M.L., X.W.); and Center for Cancer and Stem Cell Biology, Institute of Biosciences and Technology, Texas A\&M University Health Science Center, Houston, Texas (M.L.)

Received August 31, 2018; accepted November 19, 2018

\begin{abstract}
Clustered regularly interspaced short palindromic repeats (CRISPR)/ CRISPR-associated protein-9 nuclease (Cas9) technology is widely used as a tool for gene editing in rat genome site-specific engineering. Multidrug resistance 1 [MDR1 (also known as P-glycoprotein)] is a key efflux transporter that plays an important role not only in the transport of endogenous and exogenous substances, but also in tumor MDR. In this report, a novel MDR1 (Mdr1a/b) doubleknockout (KO) rat model was generated by the CRISPR/Cas9 system without any off-target effect detected. Western blot results showed that MDR1 was completely absent in the liver, small
\end{abstract}

intestine, brain, and kidney of $\mathrm{KO}$ rats. Further pharmacokinetic studies of digoxin, a typical substrate of MDR1, confirmed the deficiency of MDR1 in vivo. To determine the possible compensatory mechanism of Mdr1a/b (-/-) rats, the mRNA levels of the CYP3A subfamily and transporter-related genes were compared in the brain, liver, kidney, and small intestine of $\mathrm{KO}$ and wild-type rats. In general, a new Mdr1a/b (-/-) rat model has been successfully generated and characterized. This rat model is a useful tool for studying the function of MDR1 in drug absorption, tumor MDR, and drug target validation.

\section{Introduction}

The ATP-binding cassette (ABC) transporter family is one of the largest membrane transporter families and plays an important role in exporting endogenous and exogenous substrates from cells (Doerks et al., 2002). According to the homology of sequences and structures, the $\mathrm{ABC}$ transporter family is divided into seven subfamilies, which are sequentially numbered from A to $G$ (Dean et al., 2001). To date, 49 members of $\mathrm{ABC}$ family have been found in the human genome (Neumann et al., 2017).

Multidrug resistance (MDR) was first reported in 1970 (Biedler and Riehm, 1970). It means that once cancer cells become resistant to a certain anticancer drug, they also become resistant to other anticancer drugs. The failure of chemotherapy for most cancers is due to MDR (Li et al., 2016). Many factors can lead to MDR, especially the efflux of anticancer drugs by transporters (Gottesman et al., 2002; Leslie et al., 2005; Li et al., 2016). In ABC transporters, MDR1, also known as ABCB1 or P-glycoprotein, is most associated with MDR (Wong et al., 2015; Huang et al., 2016). MDR1 is the first discovered ABC

This work was supported in whole or part by grants from the National Natural Science Foundation of China [Grant 81773808], and the Science and Technology Commission of Shanghai Municipality [Grants 17140901000, 17140901001, and 18430760400].

${ }^{1}$ C.L., J.Z., and J.L. contributed equally to this work.

https://doi.org/10.1124/dmd.118.084277. transporter (Juliano and Ling, 1976). MDR1, a membrane protein with a molecular weight of $170 \mathrm{kDa}$, is not only expressed in many tissues, such as the small intestine, liver, kidney, and blood-brain barrier (Kimura et al., 2007; Miller et al., 2008; Zhou, 2008), but also is overexpressed in MDR cancer cell lines (Gottesman et al., 2002; Li et al., 2016).

MDR1 protein is encoded by the only gene, MDRl, in humans (Dean et al., 2001) and by two different genotypes, Mdrla and $M d r l b$, in rodents (Devault and Gros, 1990). The amino acid sequences of Mdrla and $M d r l b$ are highly overlapped, but their distribution in tissues is different. In rats, Mdrla is expressed mainly in the gastrointestinal tract, whereas $M d r 1 b$ is expressed mainly in the lung and gastrointestinal tract (Brady et al., 2002). Meanwhile, they are all expressed in the liver, kidney, and brain (Brady et al., 2002).

Gene knockout (KO) technology appeared in the 1980s and referred to the use of a certain method to delete or make inactive specific genes. Researchers can use gene KO technology to study and analyze the functions of specific genes. As early as 1994, Mdrla was knocked out in mice based on the principle of homologous recombination (Schinkel et al., 1994). In 1997, Mdrla/b (-/-) mice were successfully constructed by a previous method (Schinkel et al., 1997). Because of technical limitations, gene $\mathrm{KO}$ in rats was once restricted. According to the National Center for Biotechnology Information database, the sequence similarity of rat-human MDR1 in the gene, RNA, and protein is $81 \%, 92 \%$, and $87 \%$, respectively. In contrast, the sequence similarity

ABBREVIATIONS: ABC, ATP-binding cassette; ALB, albumin; ALP, alkaline phosphatase; ALT, alanine aminotransferase; AST, aspartate amino transferase; bp, base pair; Cas9, clustered regularly interspaced short palindromic repeats-associated protein-9 nuclease; CRISPR, clustered regularly interspaced short palindromic repeats; D-BIL, direct bilirubin; GLB, globulin; HDL-C, high-density lipoprotein-cholesterol; I-BIL, indirect bilirubin; KO, knockout; LDL-C, low-density lipoprotein-cholesterol; MDR, multidrug resistance; P450, cytochrome P450; PCR, polymerase chain reaction; SD, Sprague-Dawley; sgRNA, single guide RNA; T7E I, T7 endonuclease I; TALEN, transcription activator-like effector nuclease; TBA, total bile acid; T-BIL, total bilirubin; T-CH, total cholesterol; TG, triglyceride; TP, total protein; WT, wild type; ZFN, zinc finger nuclease. 
TABLE 1

Details for potential off-target sites examined

\begin{tabular}{|c|c|c|c|c|c|}
\hline Match Name & Location & Coordinate & Spacer + PAM & Number of Mismatches & Off-Target Score \\
\hline Mdrla_sgRNA & Chr4 & $22411028-22411008$ & AGATAGCTTTGCAAATGTAGG & & 1.000 \\
\hline Mdrla_off_1 & Chr1 & $112379519-12379501$ & ATAGCTaTGCAAATGTAG $\bar{G}$ & 1 & 0.504 \\
\hline Mdrla_off_2 & $\mathrm{Chr} 3$ & 99770595-99770575 & AGATAtaTTTGCAAAT $\overline{\text { GTGG }}$ & 2 & 0.490 \\
\hline Mdrla_off_3 & $\mathrm{ChrX}$ & 24810132-24810152 & AGATAGaTcTGCAAATGTAGG & 2 & 0.490 \\
\hline Mdrla_off_4 & Chr13 & 2551589-2551609 & AGAatGCTTTGCAAATGTt $\overline{\mathrm{GG}}$ & 2 & 0.490 \\
\hline$M d r 1 b \_s g R N A$ & Chr4 & $22303697-22303677$ & CCTCCTGATGCTGGTGTTCGG & & 1.000 \\
\hline Mdrlb_off_l & $\mathrm{Chr} 2$ & 28796708-28796688 & CCTCCTGAcGCTGGTGTTaGG & 1 & 0.700 \\
\hline Mdrlb_off_2 & $\mathrm{Chr} 7$ & 116936453-116936472 & CTCCTGcTGCTGGTGTTgGG & 1 & 0.630 \\
\hline$M d r 1 b \_o f f \_3$ & Chr18 & 32294598-32294618 & CCcCCTGAaGCTGGTGTTgGG & 2 & 0.560 \\
\hline Mdrlb_off_4 & Chr4 & 78995539-78995557 & TCCTGtTGCTGGTGTTtGG & 1 & 0.504 \\
\hline Mdrlb_off_5 & Chr9 & $72153223-72153241$ & TCCTGgTGCTGGTGTTtGG & 1 & 0.504 \\
\hline Mdrlb_off_6 & Chr12 & $31163904-31163886$ & TCCTGAaGCTGGTGTTgGG & 1 & 0.504 \\
\hline Mdrlb_off_7 & Chr7 & 25894904-25894886 & TCCTGAgGCTGGTGTTaGG & 1 & 0.504 \\
\hline
\end{tabular}

PAM, protospacer adjacent motif.

of mouse-human MDR1 in the gene, RNA, and protein is $78 \%, 85 \%$, and $78 \%$, respectively. Therefore, in the MDR1 study, the rat model is significantly better than the mouse model. In 2012, one study (Chu et al., 2012) using rat Mdrla-specific zinc finger nuclease (ZFN) established a rat model lacking the Mdrla gene. However, the function of human MDR1 protein is the sum of Mdrla and Mdr1b protein in rodents. Therefore, it is the best to use Mdrla/b (-/-) rat model to study the human MDR1 protein.

In 2013, a new gene-editing technology called clustered regularly interspaced short palindromic repeats (CRISPR)/CRISPRassociated protein-9 nuclease (Cas9) emerged (Cong et al., 2013; Mali et al., 2013). CRISPR/Cas9 system was found in bacteria and archaea as RNA-mediated adaptive immune system, which protect them from viruses and plasmids. The CRISPR/Cas9 complex consists of CRISPR RNA array, trans-activating CRISPR RNA, and Cas9 nuclease (Wang et al., 2013; Shao et al., 2014). Compared with ZFN and transcription activator-like effector nuclease (TALEN), the CRISPR/Cas9 system can edit multiple genes simultaneously and has few species limitations (Wu et al., 2013; Ceasar et al., 2016). At present, CRISPR/Cas9 technology has been widely used in animal modeling (Wang et al., 2016; Lu et al., 2017), disease research (Maresch et al., 2016), gene therapy (Guan et al., 2016; Wei et al., 2016), and other fields.

In this study, we attempted to manipulate the rat Mdrla and $M d r 1 b$ genes using CRISPR/Cas9 technology. At last, the Mdrla/b (-/-) rat model was successfully generated and then characterized for viability and physiologic status. The $M d r l a / b(-/-)$ rat can be used as a valuable tool to study the function of MDR1 in vivo.

\section{Materials and Methods}

Animals. Male and female Sprague-Dawley (SD) rats were purchased from National Rodent Laboratory Animal Resources (Shanghai, People's Republic of China). The animals were kept in a specific pathogen-free facility with access to rodent chow cubes and sterile water, with 12-hour light/dark cycles. All the

TABLE 2

Primer pairs used in this research

\begin{tabular}{|c|c|c|c|c|c|}
\hline NO & Primer Name & Primer Sequence $\left(5^{\prime}-3^{\prime}\right)$ & NO & Primer Name & Primer Sequence $\left(5^{\prime}-3^{\prime}\right)$ \\
\hline \multirow[t]{2}{*}{1} & Mdr1a-genotyping-S & GGGAAATACTCACCATCCAA & 15 & Cyp3a2-qPCR-S & AGCCTGACTTTCCCTCAA \\
\hline & Mdr1a-genotyping-A & AGCCTCCACTACATAGACCACT & & Cyp3a2-qPCR-A & TCACAGACCTTGCCAACT \\
\hline \multirow[t]{2}{*}{2} & Mdr1b-genotyping-S & GCACATCAGACGCACTTCAC & 16 & Cyp3a9-qPCR-S & CTGTGGGTTGTTAAGGGAA \\
\hline & Mdr1b-genotyping-A & CACCGCCTTTCACAGCACAA & & Сур3a9-qPCR-A & TGAGGCAGGGATCGGAGGA \\
\hline \multirow[t]{2}{*}{3} & Mdr1a-off-1-S & GGTCAAGGCTTTACTCATAT & 17 & Сур3a18-qPCR-S & ACCCAAACCTGTGCCTTTA \\
\hline & Mdr1a-off-1-A & TGTAGGACTATAAGTGGTGC & & Сyp3a18-qPCR-A & CACATGCCATCACCGTAG \\
\hline \multirow[t]{2}{*}{4} & Mdr1a-off-2-S & AAACAAGAACTTAGCCACAG & 18 & Cyp3a62-qPCR-S & AGGCAATCCTATCACCAT \\
\hline & Mdr1a-off-2-A & GTATCCCTTACAAAGCAACA & & Сур3a62-qPCR-A & GGGAATCCACGTTTACTC \\
\hline \multirow[t]{2}{*}{5} & Mdr1a-off-3-S & CTTGGGAAGCATAGCAGACA & 19 & Slc10a1-qPCR-S & AATCAAGCCTCCAAAGGACCA \\
\hline & Mdr1a-off-3-A & CCATATTCTAAGGCCCATCT & & Slc10a1-qPCR-A & GGTTTGCCCGAAGTGCTAGA \\
\hline \multirow[t]{2}{*}{6} & Mdr1a-off-4-S & GGCGTACAAAGTGACAAGAT & 20 & Slc15a1-qPCR-S & GACCAGATGCAGACGGTGAA \\
\hline & Mdr1a-off-4-A & TCAAAGGAATGAAGACTGAAAT & & Slc15a1-qPCR-A & ATTTCCGCTGGGGAAGACTG \\
\hline \multirow[t]{2}{*}{7} & Mdr1b-off-1-S & TCAAATCCACAGTGATCTGCCTAC & 21 & Slc22a1-qPCR-S & TTGTTTGGGGTTTTGGGCCT \\
\hline & Mdr1b-off-1-A & AAACGCTTCCGACTGGTGCT & & Slc22a1-qPCR-A & CCAAGTGGCAAGTCCTCCTT \\
\hline \multirow[t]{2}{*}{8} & Mdr1b-off-2-S & TTCTGCCTGGTCAAAGAGTGG & 22 & Slc28a1-qPCR-S & TGCCCATCATCATCTTCTTC \\
\hline & Mdr1b-off-2-A & AACTGCCTTCTTGTGCTTGCTT & & Slc28a1-qPCR-A & CCGCCACACTCAGTGTCTC \\
\hline \multirow[t]{2}{*}{9} & Mdr1b-off-3-S & AAGGGAAGATACCGTTCTGG & 23 & Slc1b2-qPCR-S & TGCACCTAGGTACTCTGCAT \\
\hline & Mdr1b-off-3-A & CTGAGCCATTGATCCCCACT & & Slc1b2-qPCR-A & ACCAACCCAACGAGCATCAT \\
\hline \multirow[t]{2}{*}{10} & Mdr1b-off-4-S & GGGGCTGTCCCTGTTTATCC & 24 & Abcb11-qPCR-S & TTCTTGCAAATTCCGCTGCC \\
\hline & Mdr1b-off-4-A & СССТCCTGTGAGTGCCTTTAC & & Abcb11-qPCR-A & AGACCACCCTGAAAACGTGG \\
\hline \multirow[t]{2}{*}{11} & Mdr1b-off-5-S & TTTGCCCATTGCAGCAACTT & 25 & Abcc1-qPCR-S & TGTGCAGAGGTTCTATGTGGC \\
\hline & Mdr1b-off-5-A & CCCCAGGAAGGAACGTAATAAGAG & & Abcc1-qPCR-A & TCCTCAAAGGCACGGATGAC \\
\hline \multirow[t]{2}{*}{12} & Mdr1b-off-6-S & AGTGGTCTTTGGCTAGAGTGG & 26 & Abcc2-qPCR-S & GCACATGGCTCCTGGTTTTG \\
\hline & Mdr1b-off-6-A & TGTGGTCCTGGCTATGATGC & & Abcc2-qPCR-A & ATACGCCGCATAAGACCGAG \\
\hline \multirow[t]{2}{*}{13} & Mdr1b-off-7-S & GGGAGCATGTGCCCACTATCC & 27 & Abcc3-qPCR-S & CACCATCATCCСТCAGGACCC \\
\hline & Mdr1b-off-7-A & AGAACCACCGAAGGCAGACA & & Abcc3-qPCR-A & ATCAGTCTCCAGGTCAATGGC \\
\hline \multirow[t]{2}{*}{14} & Cyp3a1-qPCR-S & CCAGCTAGAGGGACAACA & 28 & Abcg2-qPCR-S & GGGACTGATTATTGGTGCCCT \\
\hline & Сyp3a1-qPCR-A & TTATGGCACTCCACATCG & & Abcg2-qPCR-A & CACCGTCCTCTTCAGTCCTAAC \\
\hline
\end{tabular}


protocols of animal experiments in this study were approved by the Ethics Committee on Animal Experimentation of East China Normal University (Shanghai, People's Republic of China).

Chemicals and Reagents. Oligos [60 base pairs (bp), containing Mdrla or $M d r l b \mathrm{KO}$ target sites] and all primers for polymerase chain reaction (PCR) were synthesized by Biosune Biotechnology Co. LTD (Shanghai, People's Republic of China). SYBR Premix Ex Taq and Prime Script RT Reagent Kit were bought from TaKaRa (Dalian, Shanghai, People's Republic of China). Primary antibodies for MDR1 and $\beta$-actin were purchased from Abcam (Cambridge, UK). The fluorescence-conjugated secondary antibody to rabbit IgG and mouse $\mathrm{IgG}$ were bought from Cell Signaling Technology (Danvers, MA). Digoxin and digitoxin were obtained from Sigma-Aldrich (St. Louis, MO).

Target Site Selection. Selecting a right mutation target of Mdrla and Mdrlb gene is very important. In general, the first coding exon of the gene is chosen to be edited. Since the first two exon sequences of Mdrla and $M d r l b$ are less than $70 \mathrm{bp}$, the target is selected on the third exon. The selected sequences of Mdrla and $M d r l b$ genes were submitted to an online optimized CRISPR design tool (http://tools.genome-engineering.org, created by F. Zhang's laboratory at the Broad Institute of Harvard and Massachusetts Institute of Technology, Cambridge, MA) to generate some information about potential targets. The length of both the Mdrla and Mdrlb target sequences was $18 \mathrm{bp}$ followed by a protospacer adjacent motif site $\left(5^{\prime}\right.$-NGG- $\left.3^{\prime}\right)$ in the $3^{\prime}$ end.

In Vitro Construction of Templates for Transcription of Single Guide RNA and Cas9 mRNA. The first step was to synthesize a 60 bp oligodeoxynucleotides sequence, including the T7 promoter and single guide RNA (sgRNA) targets. The second step was to construct sgRNA templates by PCR with universal primers. The resulting templates can be used in vitro transcription with $\mathrm{T} 7$ transcription kit. The Cas9 plasmid was linearized by NotI, purified by phenol-chloroform extraction methods, and used as a template for in vitro transcription.

Cas9/sgRNA Injection into Zygote. Robust 8-week-old SD male rats were generally selected for vasectomy, and then 10-week-old SD female rats were selected for mating with the above-mentioned male rats for pseudopregnancy. To collect fertilized eggs, 8-week-old SD superovulated female rats were crossed with healthy fertile male rats. Embryos are obtained from superovulated female rats in the morning after mating. Before microinjection, the embryos were incubated in culture medium for $3-4$ hours at $37^{\circ} \mathrm{C}$ and $5 \% \mathrm{CO}_{2}$. Then the mixture of $M d r l a / b \operatorname{sgRNA}(25 \mathrm{ng} / \mu \mathrm{l}$ each target) and Cas 9 mRNA $(50 \mathrm{ng} / \mu \mathrm{l})$ was microinjected into the cytoplasm of one-cell embryos. The injected embryos were placed in an incubator and cultured overnight. After that, the embryos were then transplanted into the oviducts of the pseudopregnant female rats.

Genomic DNA Preparation and Genotyping. Genomic DNA was extracted from the rat toes. Purified DNA was amplified by $M d r l a / b$ primers (Table 2) with EasyTaq DNA Polymerase (TransGen Biotech Co., LTD, Beijing, People's Republic of China). To identify the genotypes of the $\mathrm{F}_{0}$ chimeras, $\mathrm{T} 7$ endonuclease I (T7E I) assay was used to determine whether the genes of the $\mathrm{F}_{0}$ generation have insertion or deletion mutations and according to the TA Cloning Kit (Thermo Fisher Scientific, Waltham, MA) instructions, PCR products amplified from each $\mathrm{F}_{0}$ genome were cloned into $\mathrm{pMD} 18-\mathrm{T}$ vectors followed by the sequencing of these clones. The genome types of $F_{1}$ and $F_{2}$ generation were identified through direct sequencing of the PCR products. The gene sequence data were analyzed by DNAMAN (Lynnon Biosoft, San Ramon, CA).

Off-Target Site Detection. The off-target information was output from the prediction website (http://cas9.wicp.net/), after the input of the target sequence of the two genes. Several sites (Table 1), with a relative high probability of being off-target (score $>0.45$ ) were selected to detect offtarget effects using the T7E I assay. Primer pairs for off-target effect detection are listed in Table 2.

Western Blot Analysis. Male Mdrla/b (-/-) rats and wild-type (WT) rats (8week-old) were sacrificed, and tissues (liver, small intestine, kidney, and brain) were collected and frozen at $-80^{\circ} \mathrm{C}$ for further processing. Tissues were ground with liquid nitrogen and then the total protein (TP) was extracted by adding radioimmunoprecipitation assay buffer to the ground tissues. Each protein sample $(100 \mu \mathrm{g} / \mathrm{lane})$ was loaded onto a $10 \%$ SDS-PAGE gel and then transferred onto the nitrocellulose membranes. Membranes were incubated with a rabbit anti-MDR1 monoclonal antibody (diluted 1:2000) at $4^{\circ} \mathrm{C}$ overnight, followed by incubation with anti-rabbit secondary antibody (diluted 1:10,000) at room temperature for 1 hour. The $\beta$-actin was used as a loading control.

Analysis of Serum Physiologic Indexes. Male Mdrla/b (-/-) rats and WT rats ( 8 weeks old) were used in this study to confirm whether there were some abnormal physiologic changes after Mdrla/b KO. Rats were fasted overnight with free access to water before serum collection. Blood samples were taken from the tail vein, and the serum samples obtained were used for clinical chemistry analysis. Serum biochemistry parameters were determined by ADICON Clinical Laboratories (Shanghai, People's Republic of China). The levels of globulin (GLB), albumin (ALB), alanine aminotransferase (ALT), aspartate amino transferase (AST), alkaline phosphatase (ALP), direct bilirubin (D-BIL), indirect bilirubin (I-BIL), total bilirubin (T-BIL), lowdensity lipoprotein-cholesterol (LDL-C), high-density lipoprotein-cholesterol (HDL-C), total cholesterol (T-CH), total bile acid (TBA), TP, and triglycerides (TGs) were detected and compared between WT and Mdrla/b (-/-) rats.

Quantitative Reverse-Transcription PCR for mRNA Analysis. The 8-weekold WT and Mdrla/b (-/-) rats were executed by carbon dioxide asphyxiation and tissues (liver, small intestine, kidney, and brain) were excised. All tissues were ground with liquid nitrogen and processed using TRIzol reagent to extract the total RNA. The total RNA was reverse transcribed into cDNA using the TaKaRa RR036A RT Kit. Real-time quantitative PCR was performed using the Quant

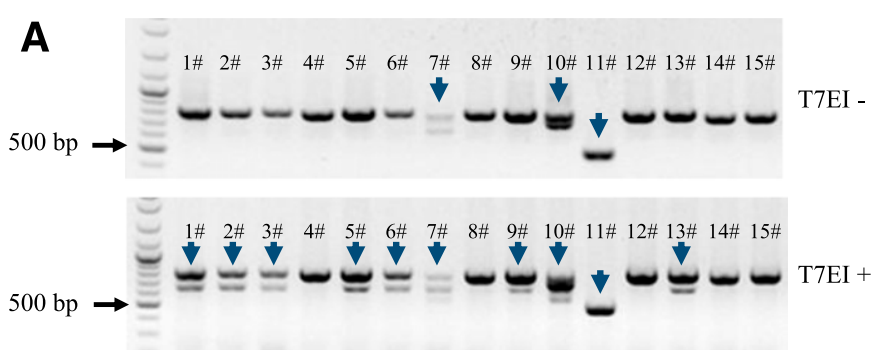

B

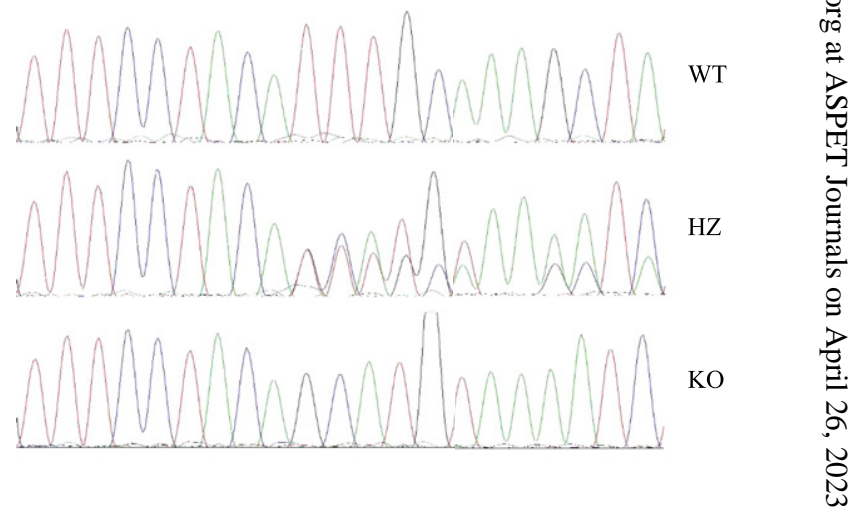

C

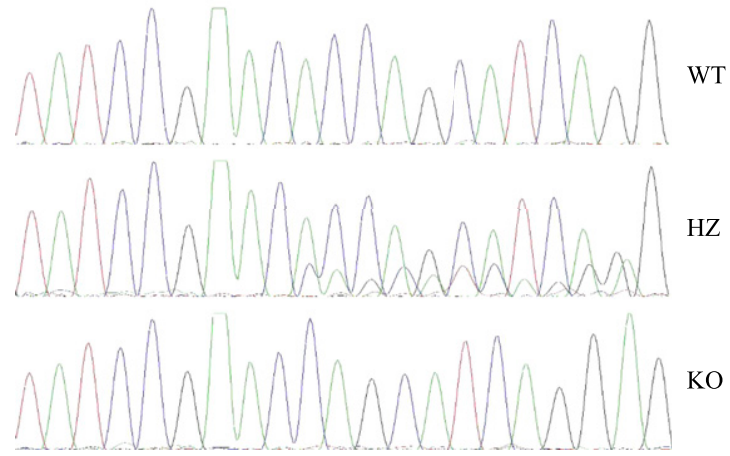

Fig. 1. Genotyping of Mdrla/b KO rats generated by the CRISPR-Cas9 system. (a) Screening offspring of gene mutations in $\mathrm{F}_{0}$ generation for Mdrla by T7E I digestion. T7E I-, before T7E I digestion; T7E I+, after T7E I digestion. The positive result indicated that there were 10 rats that may carry mutations near the Mdrla target site. (b) Sequencing peaks of Mdrla gene in WT, heterozygote, and $\mathrm{KO}$ rats in $\mathrm{F}_{2}$ generation. (c) Sequencing peaks of $M d r l b$ gene in WT, heterozygote, and $\mathrm{KO}$ rats in $\mathrm{F}_{2}$ generation. $\mathrm{HZ}$, heterozygote. 
Studio 3 Real-Time PCR System (Thermo Fisher Scientific). The relative mRNA expression of Abcb11, Abcc1, Abcc2, Abcc3, Abcg2, Slc10a1, Slc15a1, Slc1b2, Slc22al, Slc28a1, Cyp3a1, Cyp3a2, Сyp3a9, Cyp3a18, and Cyp3a62 genes were detected, and primers designed for quantification are listed in Table 2. The mRNA expression for each gene was normalized to the average of Actb in each sample.

Digoxin Pharmacokinetic Studies in WT and Mdr1a/B (-/-) Rats. Male WT and Mdrla/b (-/-) rats were used in these studies. Digoxin, evenly distributed in $0.5 \%$ CMC-Na (carboxymethyl cellulose-sodium salt), was administered orally to WT and KO rats by gavage at a single dose of $0.5 \mathrm{mg} / \mathrm{kg}$. Blood samples were collected at 5, 15, 30, 60, 120, 240, and 480 minutes from the caudal vein after digoxin administration. Blood samples were centrifuged at $5500 \mathrm{~g}$ for 15 minutes at $4^{\circ} \mathrm{C}$, and the supernatant plasma samples were transferred into new tubes and frozen at $-20^{\circ} \mathrm{C}$ until analysis.
Quantification of Digoxin in Plasma Samples by Liquid ChromatographyTandem Mass Spectroscopy. Digoxin and digitoxin (internal standard) were quantified by liquid chromatography-tandem mass spectroscopy. The system consisted of an Agilent 1290 high-performance liquid chromatography system and a 6460 Triple Quadrupole Mass Spectrometer coupled with an Agilent Jet Stream electrospray ionization ion source (Agilent Technologies, Santa Clara, CA). Chromatography separation was performed on an Agilent Zorbax Eclipse Plus C18 column $(2.1 \times 50 \mathrm{~mm}, 1.8 \mu \mathrm{m})$, using a gradient of water $(\mathrm{A})$ and acetonitrile (B) with a flow rate of $0.2 \mathrm{ml} / \mathrm{min}$. Digoxin and digitoxin were both monitored in negative electrospray ionization mode, with the ion transition of $779.3 \rightarrow 110.7$ and $763.5 \rightarrow 633.1$, respectively.

Statistical Data Analysis. The pharmacokinetic parameters of digoxin were calculated by noncompartmental modeling with WinNonlin professional

\section{A \\ T7E I \\ Mdr1a \\ Off-1}
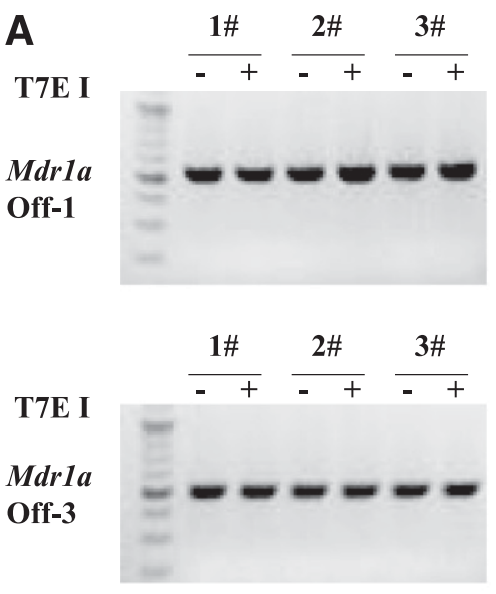

B

T7E I

Mdr1b

Off-1
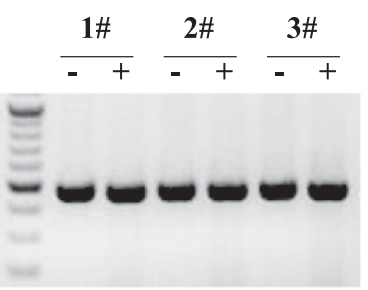

T7E I

Mdr1b

Off-3

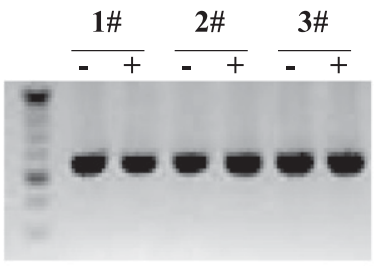

T7E I

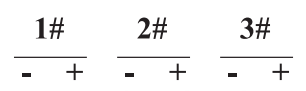

Mdr1b

Off-5

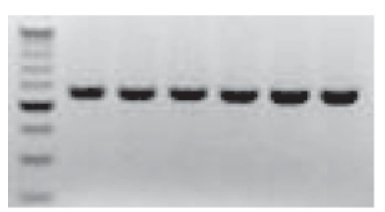

T7E I

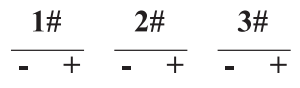

Mdr1b

Off-7
T7E I

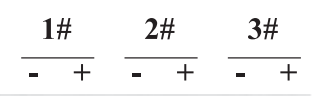

Mdr1a

Off-2

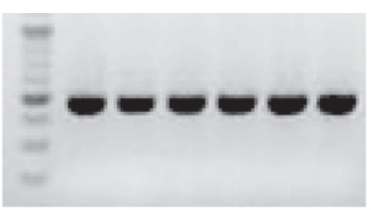

T7E I

Mdr1a

Off-4

T7E I

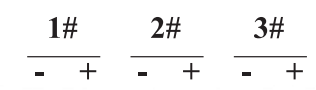

Mdr1b

Off-2
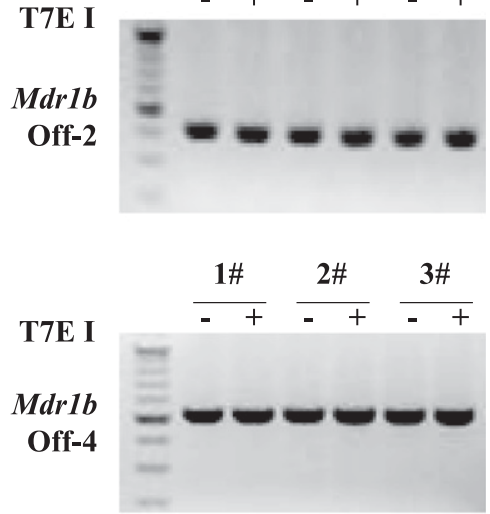

T7E I

$$
\frac{1 \#}{-+} \frac{2 \#}{-+} \frac{3 \#}{-+}
$$

Mdr1b

Off-6

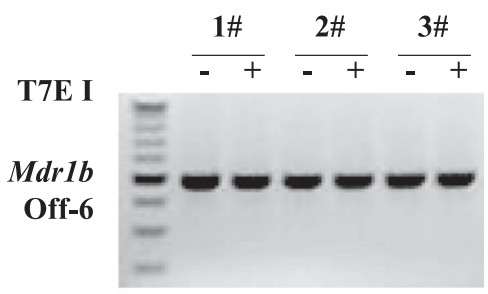

Fig. 2. Off-target detection of CRISPR/Cas9-induced mutation in three Mdrla/b KO rats. (a) Four off-target sites for Mdrla sgRNA were selected and analyzed by T7E I assay. (b) Seven off-target sites for $M d r l b$ sgRNA were selected and analyzed by T7E I assay. No off-target cleavage was detected in Mdrla/b KO rats. T7E I-, before T7E I digestion; T7E I+, after T7E I digestion. 
version 5.2.1 (Pharsight Corporation, St. Louis, MO). Mean plasma concentration-time curves were plotted by GraphPad Prism 6.0 (GraphPad Software Inc., San Diego, CA). All data were shown as the mean \pm S.D. Statistical analysis between different groups was performed using a two-tailed $t$ test, and the difference was considered to be statistically significant if the $P$ value was less than 0.05 .
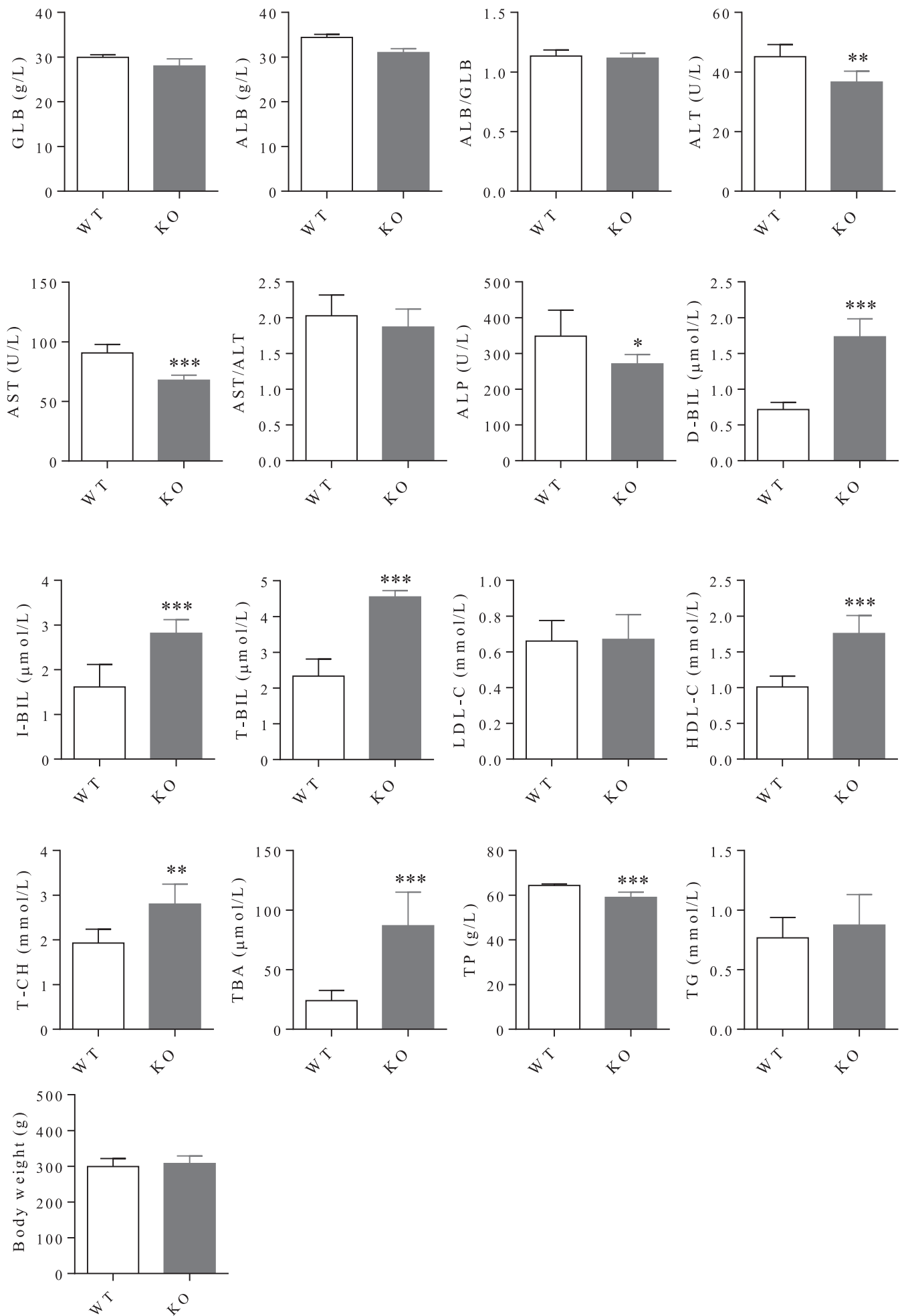

Fig. 3. Physiologic indexes of Mdrla/b double-KO rats and WT rats. Serum samples were collected from 8-week-old KO and WT male rats. The levels of GLB, ALB, ALB/AST, ALT, AST, AST/ALT, ALP, D-BIL, I-BIL, T-BIL, LDL-C, HDL-C, T-CH, TBA, TP, TGs, and body weight were calculated and compared between WT and Mdrla/b $(-/-)$ rats $(n=6)$. Values are shown as the mean \pm S.D. $* P<0.05 ; * * P<0.01 ; * * * P<0.001$ compared with WT rats. 
obtained. We randomly numbered these 15 rats of $F_{0}$ generation as $1 \#$ to 15\#. T7E I can cleave mismatched hybrid duplexes. The amplified products of $\mathrm{F}_{0}$ generation were treated with T7E I and then subjected to agarose gel electrophoresis. As shown in Fig. 1a, multiple mutant alleles were identified in these founders numbered 1\#, 2\#, 3\#, 5\#, 6\#, 7\#, 9\#, 10\#, 11\#, 13\#. Meanwhile, the electrophoresis stripes of 7\#, 10\#, and 11\# were much shorter than the expected length (795 bp) without T7E I digestion. It suggested that a relative longer gene fragment may be deleted around the target site (Fig. 1a). The 10 rats with gene mutation simultaneously amplified the sequence-targeted loci of Mdrla and $M d r 1 b$, and then the PCR products were purified and linked to the pMD18-T vectors for sequencing. The results indicated that the $F_{0}$ generations carrying both $M d r 1 a$ and $M d r l b$ mutations were 2\#, 5\#, 6\#, 7\#, 9\#, and 10\# (data not shown). Thus, these six rats were crossed with WT rats. In summary, a total of 15 young rats were born. Among them, six rats carrying both $M d r l a$ and $M d r l b$ mutations were identified, and four rats carrying only Mdrla mutations were identified. Therefore, the success rate to get $\mathrm{F}_{0}$ founders with both $M d r l a$ and $M d r l b$ mutations was $40 \%$, the success rate to get $\mathrm{F}_{0}$ founders with only Mdrla mutation was $26.7 \%$, and the success rate to get $\mathrm{F}_{0}$ founders with a mutation, including $M d r l a$ and $M d r l b$ single mutation and double mutation, was $66.7 \%$. When the $\mathrm{F}_{1}$ offspring were born, the genomes were extracted and the sequence around the target site was amplified by PCR, followed by sequencing. Only heterozygotes containing a mutation of these two genes were selected for further breeding to get homozygotes lacking both $M d r l a$ and $M d r l b$ (Fig. 1, b and c).

Off-Target Analysis. In this experiment, the sgRNA and cas9 mRNA of Mdrla and $M d r l b$ were coinjected into one-cell fertilized eggs. Thus, the possible off-target sites of two sgRNAs should be simultaneously detected in $M d r l a / b(-/-)$ rats. Three $M d r l a / b(-/-)$ rats were randomly selected to amplify the potential off-target fragments using 11 pairs designed primers (Table 2). The products were digested with T7E I and then performed by agarose gel electrophoresis. The electrophoretic bands of all products were simple and uniform in size, illustrating that analyses of rat genomic regions did not detect any offtarget cleavage (Fig. 2).

Biochemistry Analysis of WT and Mdr1a and Mdr1b Double-KO Rats. The normality of various physiologic indicators of experimental animals is a guarantee for successful experiments and reliable results. The biochemistry indexes play an important role in the establishment of experimental animal models and pharmacological and toxicological studies of drugs. Therefore, whether the gene deletion has adverse effects on rat physiology conditions, reproductive status, and liver function is a crucial step in the validation of the $M d r l a / b(-/-)$ rat model. Serum biochemistry data indicated that in $M d r l a / b(-/-)$ rats, the levels of ALB, GLB, ALB/GLB, AST/ALT, LDL-C, and TGs had no significant difference compared with those of WT rats, but the concentrations of D-BIL, I-BIL, T-BIL, HDL-C, T-CH, and TBA in KO rats were significantly increased (Fig. 3). Moreover, the levels of ALT, AST, ALP, and TP in the serum of Mdrla/b KO rats were significantly decreased, compared with those of WT rats (Fig. 3). In addition, $M d r l a / b \mathrm{KO}$ rats showed no significant and apparent differences in body weight (Fig. 3), litter size, natality, and growth/development compared with WT rats, and no sex differences were found among these characteristics.

Detection of MDR1 by Western Blot. To confirm that the CRISPRgenerated mutations resulted in an absence of MDR1 protein, we used Western blotting to detect the MDR1 expression in several tissues (liver, small intestine, kidney, and brain), in which the protein was mainly expressed. As shown in Fig. 4, the MDR1 protein was completely absent in the liver, small intestine, kidney, and brain of the three KO rats analyzed. These results proved that MDR1 was knocked out in the above-mentioned tissues.

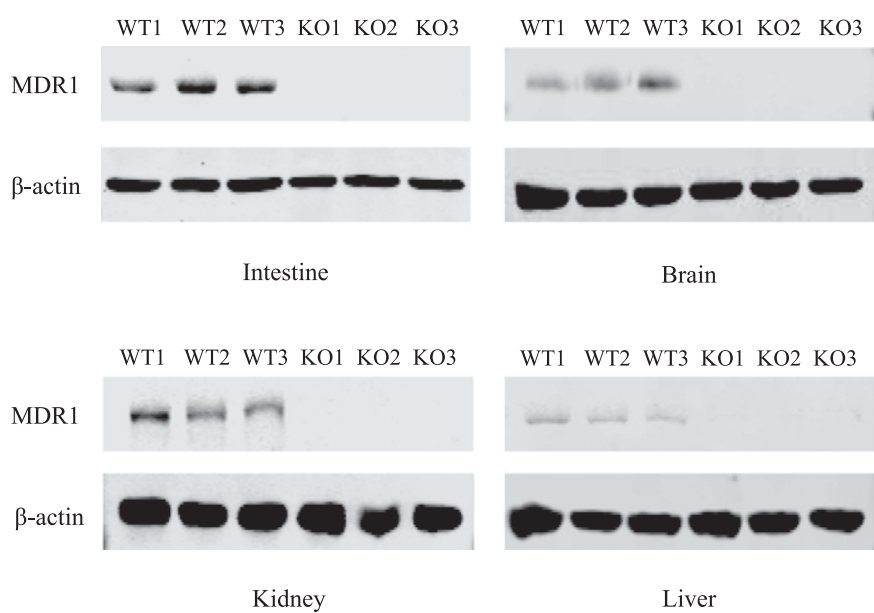

Fig. 4. Detection of MDR1 protein by Western blot. No detectable levels of MDR1 protein in intestine, brain, kidney, and liver of KO rats compared with WT rats $(n=3)$.

Compensatory Changes in Gene Expression Profiling in Liver, Small Intestine, Kidney, and Brain. To investigate whether the absence of $M d r l a$ and $M d r 1 b$ could alter the expression profile of other $\mathrm{ABC}$ transporters, solute carrier transporters and CYP3A metabolic enzymes, we used quantitative reverse-transcription PCR to determine the changes of mRNA expression in liver, small intestine, kidney, and brain of WT and $M d r l a / b(-/-)$ male rats. In small intestine, the mRNA expression of $A b c g 2, A b c c 2$, and $C y p 3 a 2$ was upregulated in $\mathrm{KO}$ rats compared with WT rats (Fig. 5a). The mRNA expression of Cyp3a62 was downregulated in the small intestine of $\mathrm{KO}$ rats, whereas the expressions of Abcc1, Abcc3, Slc15a1, Slc22al, Slc28a1, Cyp3a1, Cyp3a9, and Cyp3a18 in $\mathrm{KO}$ rats were not changed compared with WT rats (Fig. 5a). In the liver of Mdrla/b (-/-) rats, the expressions of Abcg5, Abcg8, Slc15al, Slc22a1, and Cyp3a2 were significantly lower, but Cyp3a9 expression was higher than that in WT rats (Fig. 5b). Abcc 2 mRNA and Slc15al mRNA were decreased in the kidney of Mdrla/b $(-/-)$ rats compared with WT rats (Fig. 5c). No significant change was detected in any other analyzed genes. All genes we examined in the brain of KO and WT rats showed no significant difference (Fig. 5d).

Pharmacokinetics of Digoxin in Mdr1a and Mdr1b Double-KO Rats. To further assess the damaged efflux function of MDR1 in $M d r l a / b(-/-)$ KO rats, a single dose of digoxin was administrated by gavage in both KO and WT rats. As shown in Fig. 6 and Table 3, compared with WT rats, the $C_{\max }$ and the area under the time-plasma concentration curve were significantly increased by $287 \%$ and $101 \%$ in $\mathrm{KO}$ rats, respectively. Moreover, the time to reach peak concentration of digoxin in $\mathrm{KO}$ rats was only $27 \%$ of that in WT rats, indicating that the absorption of digoxin was significantly faster in $\mathrm{KO}$ rats.

\section{Discussion}

As we all know, chemotherapy is one of the most important and effective ways to treat cancer. However, chemotherapy often does not work because of drug resistance. Among various resistance mechanisms, drug transporters are associated with MDR by extrusion of anticancer drugs (Holohan et al., 2013). In fact, at least 15 members of ABC transporter group have shown resistance to the drug (Li et al., 2016). In particular, MDR1, also known as P-glycoprotein or ABCB1, has been extensively studied in relation to MDR. MDR1 is not only expressed in many normal tissues (e.g., intestine, liver, kidney, and brain), but also is overexpressed in tumors. In addition, the expression of MDR1 can be induced by chemotherapy (Holohan et al., 2013). Since MDR1 is a key determinant of MDR, many anti-MDR strategies about MDR1 have 
A

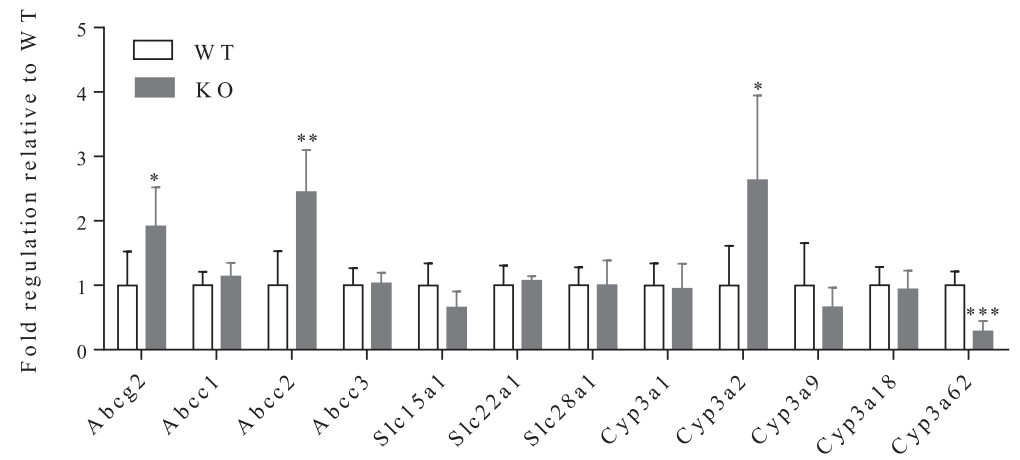

B

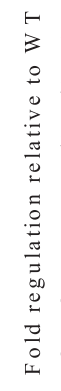

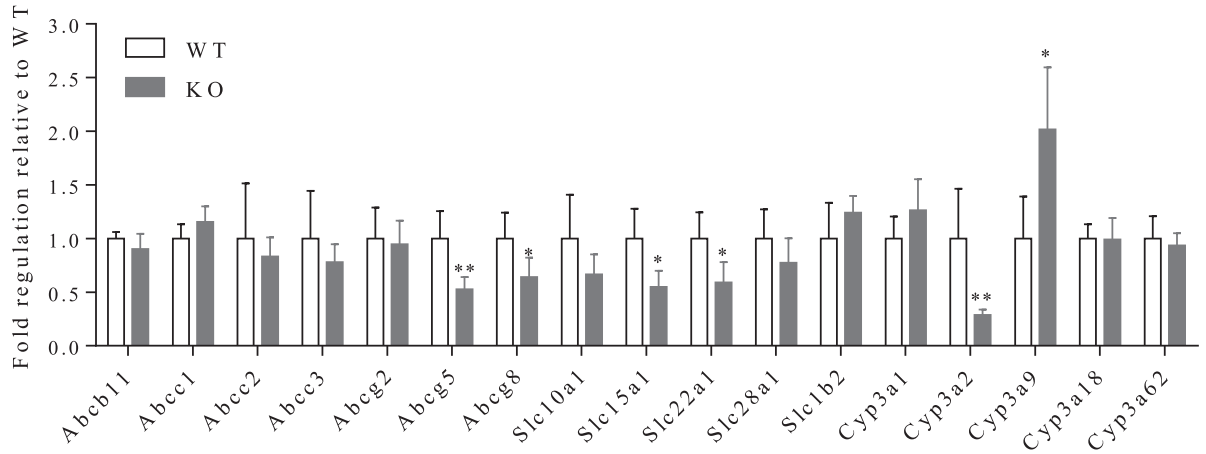

C

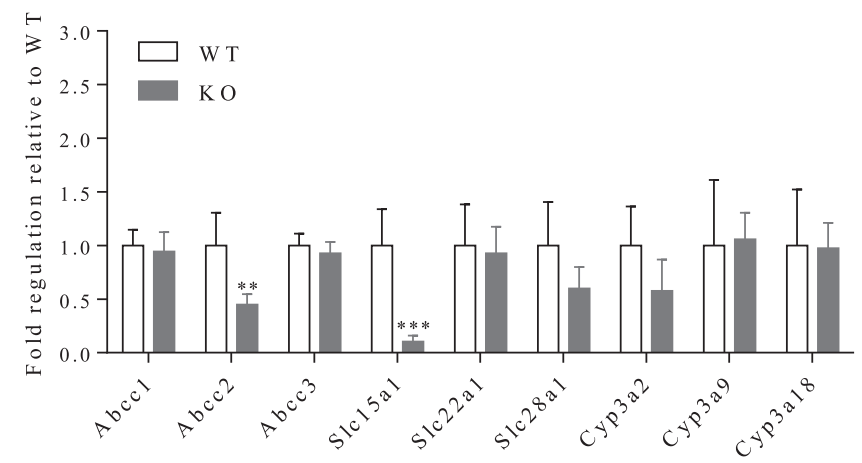

D

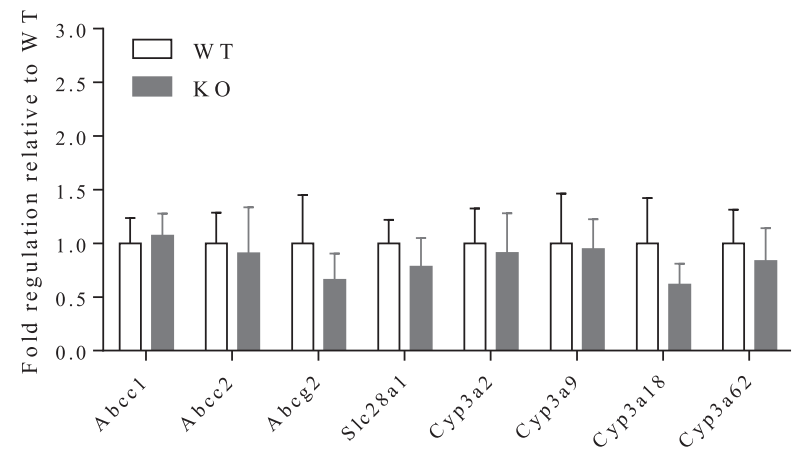

been reported (Li et al., 2016). Therefore, a novel MDR1 KO animal model is necessary to study the function of MDR1 in vivo.

The earliest gene $\mathrm{KO}$ technology is based on the principle of homologous recombination, with a low efficiency and long experimental cycle. With the development of biotechnology, the emergence of ZFN and TALEN technologies has greatly improved the efficiency of gene KO. Based on the above techniques, researchers successfully established Mdrla, Mdrlb, and Mdrla/b KO mice (Schinkel et al., 1994, 1997). However, the complexity of the experimental design and the high cost are the main constraints for further development of ZFN and TALEN. Compared with the mouse model, rats are more often used in pharmacokinetic and toxicological studies because of, for example, their
Fig. 5. Gene expression of various transporters and $C y p 3 a$ subfamily in Mdrla/b (-/-) rat intestine (a), liver (b), kidney (c), and brain (d) compared with WT rats, which were analyzed using quantitative PCR $(n=5)$. Values are shown as the mean \pm S.D. $* P<0.05 ; * * P<$ $0.01 ; * * * P<0.001$ compared with WT rats. large size and adequate blood volume. In particular, according to the data in the National Center for Biotechnology Information database, MDR1 in rats is closer to humans in terms of genome level, RNA level, and protein level. Therefore, rats are significantly better than mice in the study of MDR1. Although the Mdrla gene KO was reported by the ZFN technique in rats (Chu et al., 2012), the $M d r 1 a / b$ double-KO rat model did not appear because of the difficulty of gene editing. This study successfully generated the Mdrla/b KO rat model based on CRISPR/ Cas9 technology for the first time. Western blot assay confirmed that there was no MDR1 in the small intestine, brain, kidney, and liver. Further, the loss of MDR1 function significantly increased digoxin uptake in $M d r l a / b(-/-)$ rats. 


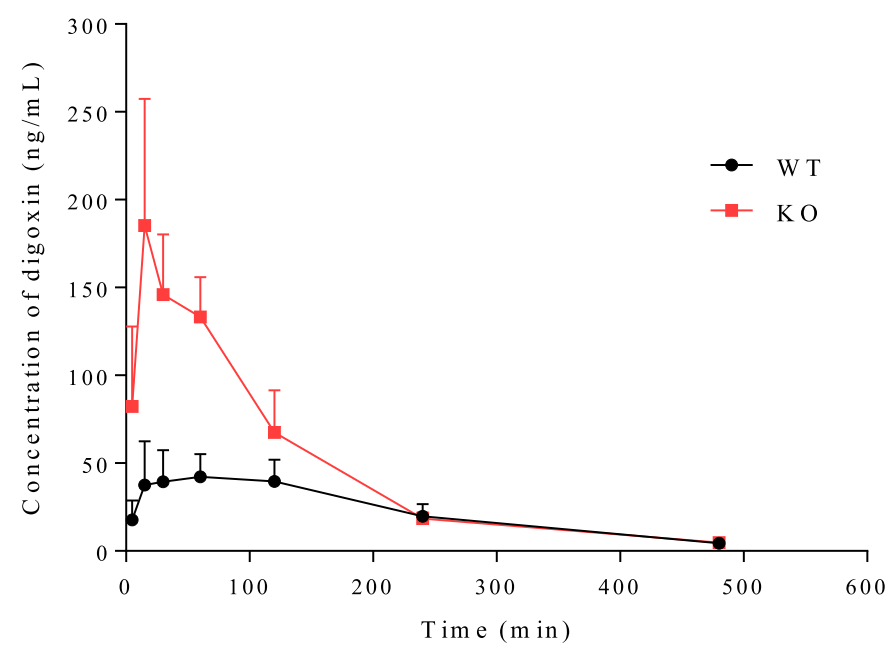

Fig. 6. Plasma concentrations of digoxin in KO and WT rats were measured by liquid chromatography-tandem mass spectroscopy after oral administration $(0.5 \mathrm{mg} / \mathrm{kg})$ of digoxin. Each value represents the mean \pm S.D. of six rats.

The normal physiologic function of laboratory animals is an important prerequisite and is a guarantee for reliable experimental results. Physiologic indexes of experimental animals are also an important part of establishing experimental animal models in pharmacology and toxicology research. Therefore, it is an important step to make sure of the adverse effects on various physiologic indexes in the validation of the $M d r l a / b(-/-)$ rat model. By comparing the serum clinical chemistry parameters of adult $\mathrm{KO}$ and WT rats at the same age, we found that $\mathrm{KO}$ and WT rats had equivalent levels of ALB, GLB, LDL-C, TG, TP, ALB/GLB, and AST/ALT. However, the serum levels of D-BIL, I-BIL, T-BIL, HDL-C, T-CH, and TBA were significantly upregulated in $M d r l a / b(-/-)$ rats. Some experimental studies have shown that the presence of cholesterol increases the rate of MDR1 drug efflux (Sheehy et al., 2015; Subramanian et al., 2016). Cholesterol is catalyzed by CYP7A1, and through a series of biochemical reactions it eventually produces cholic acid, which is a primary bile acid (Dawson, 2015). Bile acid and cholesterol are secreted into the bile duct through ABCB11 and ABCG5/G8 (Yu et al., 2002; Stieger, 2009). Previous reports (Wang et al., 2009) have claimed that MDR1 and ABCB11 play a compensatory role in bile salt transport. In this study, the expression of $A b c g 5$ and $A b c g 8$ in the liver of $\mathrm{KO}$ rats was significantly lower than that of WT rats. Therefore, when MDR1 is null, cholesterol and bile acids may be deposited in the body. Previous studies (Bellarosa et al., 2009) have reported that MDR1 may limit the transfer of bilirubin through the blood-brain barrier, suggesting that bilirubin may be an endogenous substrate of MDR1. We also found that Mdrla-deficient rats accumulated higher levels of D-BIL, I-BIL, and T-BIL than WT rats. However, in Mdrla/b (-/-) rats, the levels of ALP, AST, and ALT were significantly downregulated, whereas the AST/ALT level in serum was not significant different compared with WT rats. These changes may not cause liver parenchymal damage, but these changes in clinical chemistry may lead to physiologic differences between Mdrla/b (-/-) and WT rats, which should be considered in the further application of this model.

To investigate whether the deletion of Mdrla and Mdrlb alters the expression profiles of other drug transporters, we selected some important solute carrier transporters and other ABC transporters associated with drug transport, and then detected their mRNA expression in the major expressed tissues. The important $A B C$ proteins include MDR 1 (MDR1, gene symbol $A B C B 1$ ), MDR protein 1 (MRP1, gene symbol $A B C C 1$ ), MDR protein 2 (MRP2, gene symbol $A B C C 2$ ), and breast cancer resistance protein (BCRP, gene symbol $A B C G 2)$. Our data
TABLE 3

Pharmacokinetic parameters of digoxin after oral administration in WT and KO rats

The dose of digoxin was $0.5 \mathrm{mg} / \mathrm{kg}$. All data are shown as the mean $\pm S . D .(n=6)$.

\begin{tabular}{lcc}
\hline \multicolumn{1}{c}{ Parameters } & WT & KO \\
\hline$C_{\max }(\mathrm{ng} / \mathrm{ml})$ & $49.9 \pm 20.3$ & $193.4 \pm 65.5^{* * * *}$ \\
$\mathrm{t}_{\max }(\mathrm{min})$ & $82.5 \pm 44.2$ & $22.5 \pm 18.4^{*}$ \\
$\mathrm{AUC}_{0-\mathrm{t}}(\mathrm{ng} / \mathrm{min}$ per milliliter $)$ & $11,033.3 \pm 2938.1$ & $22,182.8 \pm 3658.5^{* * *}$ \\
$\mathrm{AUC}_{0-\infty}(\mathrm{ng} / \mathrm{min}$ per milliliter $)$ & $11,827.9 \pm 2967.3$ & $22,831.2 \pm 3646.2^{* * *}$ \\
\hline
\end{tabular}

$\mathrm{AUC}_{0-\infty}$, area under the plasma concentration-time curve during $0-\infty \mathrm{min} ; \mathrm{AUC}_{0-\mathrm{t}}$, area under the plasma concentration-time curve during $0-480 \mathrm{~min} ; \mathrm{t}_{\max }$, time to peak plasma concentration. $* P<0.05 ; * * * P<0.001$ compared with WT rats.

showed that $A b c g 2$ was upregulated only in the intestinal tract of male $M d r l a / b(-/-)$ rats. The expression of $A b c c 2$ in intestinal tissues was significantly increased, whereas the expression of $A b c c 2$ in renal tissues of male $M d r l a / b(-/-)$ rats was significantly decreased. Substantial evidence suggests that these efflux pumps have overlapping functions in tissue defense and MDR (Leslie et al., 2005). The intestine represents the first barrier of the body and is a defense against the invasion of xenobiotics including toxins and drugs. After KO MDR1, this defensive ability may be weakened, and other transporters will also be upregulated. However, it is not clear whether this change at the mRNA level will lead to changes in protein expression, which needs further study.

Cytochrome P450 (P450), a heme-containing superoxide monooxygenase superfamily, plays an important role in the metabolism of various endogenous and exogenous substances. P450 is one of the most important phase I drug-metabolizing enzymes in the human body, and is expressed mainly in the liver, gastrointestinal tract, and kidney, with a small amount of expression in the brain, lung, breast, skin, and other organs and tissues (Estabrook, 2003). P450 enzymes involved in drug metabolism are mainly in the CYP1/2/3 families. The CYP3A subfamily, one of the most metabolically active subtypes, plays a key role in eliminating endogenous substances and more than half of therapeutic drugs (Takara et al., 2003). In rats, CYP3A subtype has five members: CYP3A1, CYP3A2, CYP3A9, CYP3A18, and CYP3A62 (Takara et al., 2003; Matsubara et al., 2004). These isoforms are predominantly expressed in the liver, but can also be found in other organs such as the intestine, kidney, and brain. In fact, the distribution of CYP3A and MDR1 is very similar. More interestingly, there is a wide overlap between the substrates of CYP3A and MDR1 (van Waterschoot and Schinkel, 2011). Recent studies (van Waterschoot and Schinkel, 2011; $\mathrm{Yu}$ et al., 2014) have reported that CYP3A and MDR1 have similar functions in regulating oral drug bioavailability, drug resistance, and drug-drug interaction. This study found that Cyp3a62 expression was significantly lower in the intestine and Cyp $3 a 9$ expression was much higher in the liver of male $M d r l a / b(-/-)$ rats. Cyp3a2 expression was hiked in the intestine but lower in the liver of male $M d r l a / b(-/-)$ rats. There were no significant changes in the expression of other Сyp3a subtypes at the mRNA level in $M d r l a / b(-/-)$ rats. The strategic colocalization of CYP3A and MDR1 in intestinal epithelial cells and hepatocytes leads to the possible interaction of these proteins in the treatment of common substrates. The specific mechanism of this change is still unclear. Further studies are needed to confirm whether these changes can affect CYP3A activity.

Digoxin, recommended by the Food and Drug Administration, is often used as a probe substrate for MDR1 in vivo pharmacokinetic studies (Nader and Foster, 2014), because it has the advantage of not being metabolized by any CYP3A enzyme (Lacarelle et al., 1991; Fenner et al., 2009). In this study, the significant difference in digoxin absorption between WT and KO rats was observed, thus suggesting that the Mdrla/b double-KO rat can be used as a sensitive model to study the activity of MDR 1 in vivo. In addition, 
the $M d r l a / b$ double-KO rat could be used to evaluate whether compounds are substrates for MDR1.

In summary, an $M d r l a / b \mathrm{KO}$ rat model was successfully generated using CRISPR/Cas9 technology. No off-target cleavage was detected at 11 predicted sites of the $M d r l a / b \mathrm{KO}$ rat. This new rat model is of great significance to further investigate the function of MDR1 in drug transport, toxicity, and drug resistance. Moreover, this model may be a useful tool for studying the partnership between MDR1 and CYP3A in vivo.

\section{Authorship Contributions}

Particiipated in research design: $\mathrm{Xu}$. Ma, Liu, and Wang.

Conducted experiments: Liang, Zhao, Lu, Zhang, Xi. Ma, Shang, and Li.

Performed data analysis: Liang, Zhao, Lu, Zhang, Xi. Ma, Li, and Wang.

Wrote or contributed to the writing of the manuscript: Liang, Zhao, Lu, Zhang, Xi. Ma, Shang, Xu. Ma, Liu, and Wang.

\section{References}

Bellarosa C, Bortolussi G, and Tiribelli C (2009) The role of ABC transporters in protecting cells from bilirubin toxicity. Curr Pharm Des 15:2884-2892.

Biedler JL and Riehm H (1970) Cellular resistance to actinomycin D in Chinese hamster cells in vitro: cross-resistance, radioautographic, and cytogenetic studies. Cancer Res 30: 1174-1184.

Brady JM, Cherrington NJ, Hartley DP, Buist SC, Li N, and Klaassen CD (2002) Tissue distribution and chemical induction of multiple drug resistance genes in rats. Drug Metab Dispos 30: 838-844.

Ceasar SA, Rajan V, Prykhozhij SV, Berman JN, and Ignacimuthu S (2016) Insert, remove or replace: a highly advanced genome editing system using CRISPR/Cas9. Biochim Biophys Acto 1863:2333-2344.

Chu X, Zhang Z, Yabut J, Horwitz S, Levorse J, Li XQ, Zhu L, Lederman H, Ortiga R, Strauss J, et al. (2012) Characterization of multidrug resistance $1 \mathrm{a} / \mathrm{P}$-glycoprotein knockout rats generated by zinc finger nucleases. Mol Pharmacol 81:220-227.

Cong L, Ran FA, Cox D, Lin S, Barretto R, Habib N, Hsu PD, Wu X, Jiang W, Marraffini LA, et al. (2013) Multiplex genome engineering using CRISPR/Cas systems. Science 339:819-823.

Dawson PA (2015) Impact of inhibiting ileal apical versus basolateral bile acid transport on cholesterol metabolism and atherosclerosis in mice. Dig Dis 33:382-387.

Dean M, Rzhetsky A, and Allikmets R (2001) The human ATP-binding cassette (ABC) transporter superfamily. Genome Res 11:1156-1166.

Devault A and Gros P (1990) Two members of the mouse mdr gene family confer multidrug resistance with overlapping but distinct drug specificities. Mol Cell Biol 10:1652-1663.

Doerks T, Copley RR, Schultz J, Ponting CP, and Bork P (2002) Systematic identification of novel protein domain families associated with nuclear functions. Genome Res 12:47-56.

Estabrook RW (2003) A passion for P450s (rememberances of the early history of research on cytochrome P450). Drug Metab Dispos 31:1461-1473.

Fenner KS, Troutman MD, Kempshall S, Cook JA, Ware JA, Smith DA, and Lee CA (2009) Drugdrug interactions mediated through P-glycoprotein: clinical relevance and in vitro-in vivo correlation using digoxin as a probe drug. Clin Pharmacol Ther 85:173-181.

Gottesman MM, Fojo T, and Bates SE (2002) Multidrug resistance in cancer: role of ATPdependent transporters. Nat Rev Cancer 2:48-58.

Guan Y, Ma Y, Li Q, Sun Z, Ma L, Wu L, Wang L, Zeng L, Shao Y, Chen Y, et al. (2016) CRISPR/Cas9-mediated somatic correction of a novel coagulator factor IX gene mutation ameliorates hemophilia in mouse. EMBO Mol Med 8:477-488.

Holohan C, Van Schaeybroeck S, Longley DB, and Johnston PG (2013) Cancer drug resistance: an evolving paradigm. Nat Rev Cancer 13:714-726.

Huang QT, Shynlova O, Kibschull M, Zhong M, Yu YH, Matthews SG, and Lye SJ (2016) Pglycoprotein expression and localization in the rat uterus throughout gestation and labor. Reproduction 152:195-204.

Juliano RL and Ling V (1976) A surface glycoprotein modulating drug permeability in Chinese hamster ovary cell mutants. Biochim Biophys Acta 455:152-162.

Kimura Y, Morita SY, Matsuo M, and Ueda K (2007) Mechanism of multidrug recognition by MDR1/ABCB1. Cancer Sci 98:1303-1310.

Lacarelle B, Rahmani R, de Sousa G, Durand A, Placidi M, and Cano JP (1991) Metabolism of digoxin, digoxigenin digitoxosides and digoxigenin in human hepatocytes and liver microsomes. Fundam Clin Pharmacol 5:567-582.

Leslie EM, Deeley RG, and Cole SP (2005) Multidrug resistance proteins: role of P-glycoprotein, MRP1, MRP2, and BCRP (ABCG2) in tissue defense. Toxicol App Pharmacol 204:216-237.
Li W, Zhang H, Assaraf YG, Zhao K, Xu X, Xie J, Yang DH, and Chen ZS (2016) Overcoming $\mathrm{ABC}$ transporter-mediated multidrug resistance: molecular mechanisms and novel therapeutic drug strategies. Drug Resist Updat 27:14-29.

Lu J, Shao Y, Qin X, Liu D, Chen A, Li D, Liu M, and Wang X (2017) CRISPR knockout rat cytochrome P450 3A1/2 model for advancing drug metabolism and pharmacokinetics research. Sci Rep 7:42922.

Mali P, Yang L, Esvelt KM, Aach J, Guell M, DiCarlo JE, Norville JE, and Church GM (2013) RNA-guided human genome engineering via Cas9. Science 339:823-826.

Maresch R, Mueller S, Veltkamp C, Öllinger R, Friedrich M, Heid I, Steiger K, Weber J, Engleitner T, Barenboim M, et al. (2016) Multiplexed pancreatic genome engineering and cancer induction by transfection-based CRISPR/Cas9 delivery in mice. Nat Commun 7:10770.

Matsubara T, Kim HJ, Miyata M, Shimada M, Nagata K, and Yamazoe Y (2004) Isolation and characterization of a new major intestinal CYP3A form, CYP3A62, in the rat. J Pharmacol Exp Ther 309: 1282-1290.

Miller DS, Bauer B, and Hartz AM (2008) Modulation of P-glycoprotein at the blood-brain barrier: opportunities to improve central nervous system pharmacotherapy. Pharmacol Rev 60:196-209.

Nader AM and Foster DR (2014) Suitability of digoxin as a P-glycoprotein probe: implications of other transporters on sensitivity and specificity. J Clin Pharmacol 54:3-13.

Neumann J, Rose-Sperling D, and Hellmich UA (2017) Diverse relations between ABC transporters and lipids: an overview. Biochim Biophys Acta Biomembr 1859:605-618.

Schinkel AH, Mayer U, Wagenaar E, Mol CA, van Deemter L, Smit JJ, van der Valk MA, Voordouw AC, Spits H, van Tellingen O, et al. (1997) Normal viability and altered pharmacokinetics in mice lacking mdr1-type (drug-transporting) P-glycoproteins. Proc Natl Acad Sci USA 94:4028-4033.

Schinkel AH, Smit JJ, van Tellingen O, Beijnen JH, Wagenaar E, van Deemter L, Mol CA, van der Valk MA, Robanus-Maandag EC, te Riele HP, et al. (1994) Disruption of the mouse mdr1a P-glycoprotein gene leads to a deficiency in the blood-brain barrier and to increased sensitivity to drugs. Cell 77:491-502.

Shao Y, Guan Y, Wang L, Qiu Z, Liu M, Chen Y, Wu L, Li Y, Ma X, Liu M, et al. (2014) CRISPR/Cas-mediated genome editing in the rat via direct injection of one-cell embryos. Nat Protoc 9:2493-2512.

Sheehy RM, Kuder CH, Bachman Z, and Hohl RJ (2015) Calcium and P-glycoprotein independent synergism between schweinfurthins and verapamil. Cancer Biol Ther 16:1259-1268.

Stieger B (2009) Recent insights into the function and regulation of the bile salt export pump (ABCB11). Curr Opin Lipidol 20:176-181.

Subramanian N, Schumann-Gillett A, Mark AE, and O'Mara ML (2016) Understanding the accumulation of P-glycoprotein substrates within cells: the effect of cholesterol on membrane partitioning. Biochim Biophys Acta 1858:776-782.

Takara K, Ohnishi N, Horibe S, and Yokoyama T (2003) Expression profiles of drug-metabolizing enzyme CYP3A and drug efflux transporter multidrug resistance 1 subfamily mRNAS in small intestine. Drug Metab Dispos 31:1235-1239.

van Waterschoot RA and Schinkel AH (2011) A critical analysis of the interplay between cytochrome P450 3A and P-glycoprotein: recent insights from knockout and transgenic mice. Pharmacol Rev 63:390-410

Wang H, Yang H, Shivalila CS, Dawlaty MM, Cheng AW, Zhang F, and Jaenisch R (2013) Onestep generation of mice carrying mutations in multiple genes by CRISPR/Cas-mediated genome engineering. Cell 153:910-918.

Wang R, Chen HL, Liu L, Sheps JA, Phillips MJ, and Ling V (2009) Compensatory role of P-glycoproteins in knockout mice lacking the bile salt export pump. Hepatology 50:948-956.

Wang X, Tang Y, Lu J, Shao Y, Qin X, Li Y, Wang L, Li D, and Liu M (2016) Characterization of novel cytochrome P450 2E1 knockout rat model generated by CRISPR/Cas9. Biochem Phar macol 105:80-90.

Wei Y, Chen Y, Qiu Y, Zhao H, Liu G, Zhang Y, Meng Q, Wu G, Chen Y, Cai X, et al. (2016) Prevention of muscle wasting by CRISPR/Cas9-mediated disruption of myostatin in vivo. Mo Ther 24:1889-1891.

Wong IL, Wang BC, Yuan J, Duan LX, Liu Z, Liu T, Li XM, Hu X, Zhang XY, Jiang T, et al (2015) Potent and nontoxic chemosensitizer of P-glycoprotein-mediated multidrug resistance in cancer: synthesis and evaluation of methylated epigallocatechin, gallocatechin, and dihydromyricetin derivatives. J Med Chem 58:4529-4549.

Wu Y, Liang D, Wang Y, Bai M, Tang W, Bao S, Yan Z, Li D, and Li J (2013) Correction of a genetic disease in mouse via use of CRISPR-Cas9. Cell Stem Cell 13:659-662.

Yu CP, Hsieh YW, Lin SP, Chi YC, Hariharan P, Chao PD, and Hou YC (2014) Potentia modulation on P-glycoprotein and CYP3A by soymilk and miso: in vivo and ex-vivo studies. Food Chem 149:25-30.

Yu L, Li-Hawkins J, Hammer RE, Berge KE, Horton JD, Cohen JC, and Hobbs HH (2002) Overexpression of ABCG5 and ABCG8 promotes biliary cholesterol secretion and reduces fractional absorption of dietary cholesterol. J Clin Invest 110:671-680.

Zhou SF (2008) Structure, function and regulation of P-glycoprotein and its clinical relevance in drug disposition. Xenobiotica 38:802-832.

Address correspondence to: Dr. Xin Wang, East China Normal University, Dongchuan Road 500, Shanghai 200241, People's Republic of China. E-mail: xwang@bio.ecnu.edu.cn; usxinwang@gmail.com 